\begin{tabular}{l|l|l} 
Volume (26) - Issue (2) - 2018 & ISSN:1110-256X \\
& DOI: 10.21608/joems.2018.2671.1021
\end{tabular}

\title{
AUTOMATIC PLANT RECOGNITION AND DISEASES IDENTIFICATION METHODS BASED ON IMAGE PROCESSING TECHNIQUES
}

\author{
Ashraf Darwish \\ Faculty of Science, Helwan University, Cairo, Egypt \\ ashraf.darwish.eg@iee.org
}

Received 17/1/2018

Revised

Accepted 24/3/2018

\begin{abstract}
Plant recognition and diseases identification have an impact on the sustainable development of many countries in the agricultural sector. The automatic plant recognition and diseases identification will assist the specialists and experts in agriculture to overcome many of plant diseases and problems. The automation of plant diseases identification and recognition approaches have received considerable interest in the last years because their effect on the growth of the economy of countries, which may depend mainly on agriculture and to reduce the economic losses in the sustainable agriculture industry in general. However, human cognition and sight are not sufficient to identify the region of interest in the images of plants, usually, stems and leaves. Nowadays, image-based methods are considered as a visual assisting of plant recognition and diseases identification with the aid of the recent advances in image processing area. In this paper, we describe and analyze the automated image-based methods and discuss the state-of-art of plant recognition and diseases identification that has been applied in the last years. Also, we explore the role of image processing methods and classifiers in plant diseases identification and recognition. Different types of datasets of plant diseases identification and recognition are introduced briefly with their existing problems. As an example, the preprocessing phase of this issue is implemented based on real infected tomato leaves. Also, shape feature, color feature, and texture feature have been reviewed. Moreover, we described the important classifiers that are used currently used in the classification process. Also, hybrid classifiers can integrate the results from multiple algorithms with the aim of improving classification accuracy. Therefore, some of the well-known hybrid classifiers for plant diseases identification and recognition have been presented. Some solutions of using image-based methods such as complex backgrounds of the region of interest, different plant diseases can produce similar symptoms, and the conditions of capturing images have been presented. Finally, some points of the future work are proposed.

Keywords: Plant identification, Plant diseases, image processing, classifiers, feature extraction, shape feature, color feature, texture feature.

Mathematics Subject Classification: 68, 93, 94

\section{Introduction and Related Work}

Plant industry is representing the core of the growth of many countries. Usually, each plant has specific characteristics such as habits, morphology, and economic value. There are many kinds of plants worldwide which had been recorded and named by the statistics as in [1]. To make this industry effective, we apply image processing techniques to the study on plant recognition and identification. The developments in image processing area lead to used new methods and techniques in plant recognition diseases identification. With the advancements in digital pattern recognition and image processing methods, it will provide new opportunities for plant recognition and diseases identification. In the last decades, researchers gained an interest in plant diseases identification. Therefore, in the last years, researchers became are interested in plant diseases identification for their importance and impact on the future of agriculture in adopting image processing techniques. However, plant diseases identification represents a new challenge in machine learning. The challenge in this process is the similarity in plant species which are very similar to each other. Also, plant maturity changes can lead to the large variation within one species.

The plant image identification issue was addressed as a track in the CLEF international conference as in [2]. In the literature, some systems had been developed for plant identification problems. For example, plant images for
\end{abstract}


identification and classification tasks as in $[3,4]$. The richness of plant color is an important factor in identifying the diseases. Therefore it is useful to depend on flowers plants for the identification process. In [5] different models of color are used for the segmentation of flowers. According to [6], there are a number of fully automatic plant identification systems in ImageCLEF'2012 that showed high efficiency in plant diseases diagnosis, LSIS-DYNI-run3 (LSIS - (laboratory des Sciences de l'Information et des Systèmes (http://www.lsis.org/), DYNI (Université du Sud Toulon-Var, BP 20132, 83957 La Garde, France)), multiple classifications of plant leaves based on Gabor transform and LBP operator are proposed in [7], and IFSC/USP (IFSC (Instituto de Física de São Carlos (https://www2.ifsc.usp.br/english/) ) [8]. Figure 1 describes the plant diseases identification based on image processin two class problemg techniques since 2000 to 2016 based on statistics of the web of knowledge databases.

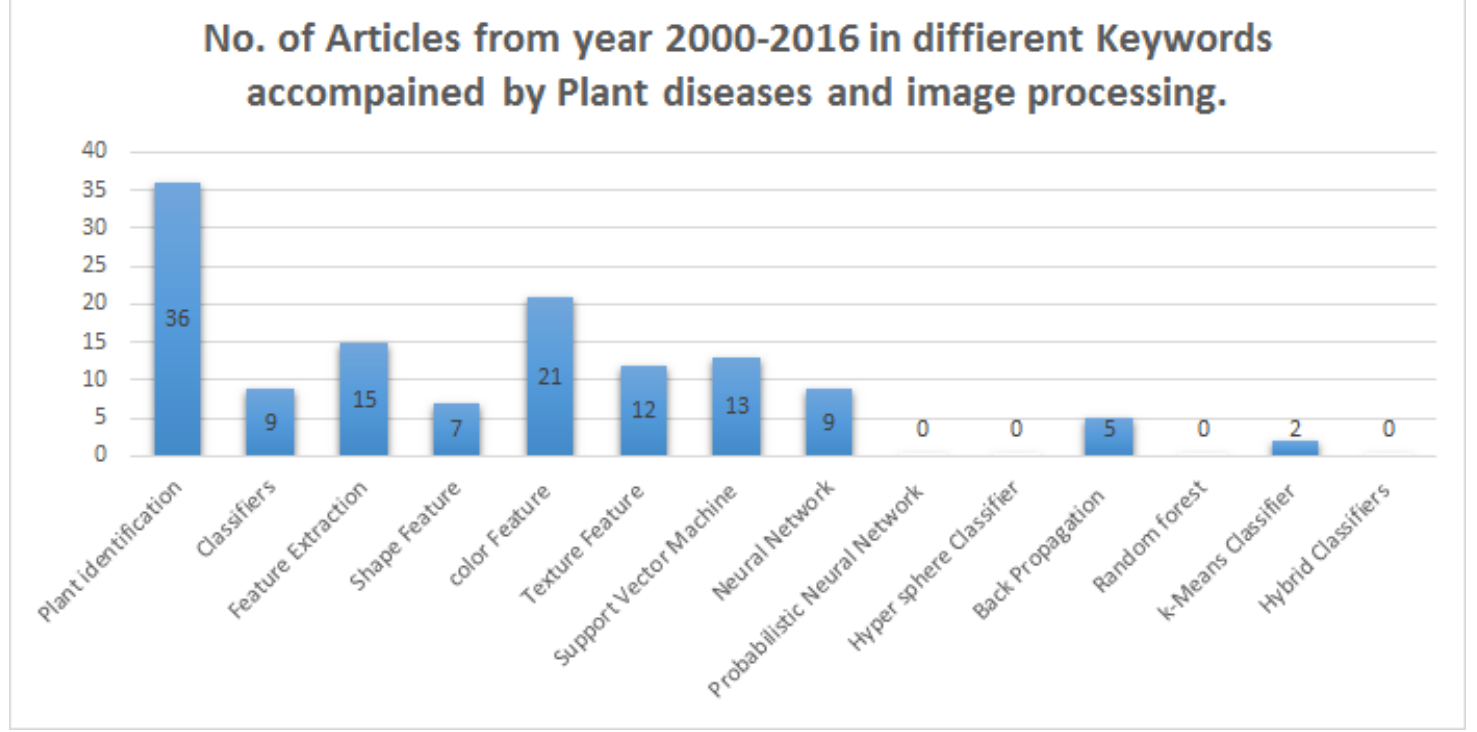

Fig.1. Distribution of plant diseases identification from 2000-2016 according to Web of Science with some related keywords.

The remaining of this paper is as follows. Section II presents the framework of plant recognition and diseases identification, and some segmentations approaches are introduced. Section III explores the feature extraction methods base on color, shape, and texture features. The commonly used classifiers in the literature are presented in section IV. Section V presents the existing problems, limitations, and challenges in plant recognition and diseases identification. Finally, the conclusion and the future work of this paper are described in section VI.

\section{Image Processing Methods for Plant Identification Diseases}

\section{Traditional Methods of Plant Diseases Identification}

The plant detections methods can be classified into two methods indirect and direct methods. In traditional methods, there are serological (enzyme-linked immunosorbent assay, flow cytometer and molecular (fluorescence in situ hybridization and DNA arrays). On the other hand, indirect methods include plant metabolite profiling, biomarkerbased detection of disease, fluorescence, and hyper spectral imaging techniques, and infrared RFID, sensors techniques. Although the availability of such methods is still needed for low-cost methods, fast, and sensitive methods to identify the diseases of plants. There are many automatic systems based on image processing for plant diseases identification which will play an important role shortly in the protection of plant industry.

\section{Why Computer-based images}

Plants are an essential resource for foods to human especially with the increasing number of population and climate change worldwide. Therefore, there is an interest to find new solutions based on the advances in computational methods and techniques of an image processing to identify the plant diseases. The demand for identification of plant with the help of computer vision and image processing has increasingly used in the last years to develop economies of countries and to reduce the labor cost. In this regard, researchers developed a new automated system to identify the plant diseases with a high level of accuracy. In the botany science, leaves of plants are representing an interest of researchers because they can assist in differentiating different species of plant to identify the diseases. The classification process is playing a crucial role in identifying plant diseases. With the developments in digital cameras, this will increase demand for providing datasets of plant images that can be processed by image processing techniques. Also, plant modeling is a complementary process in plant recognition and diseases identification process. In the 
literature, there different algorithms that have been developed to reproduce the branching structure of plants as described in [9]. The different phases of plant identification process are described in Figure 2.

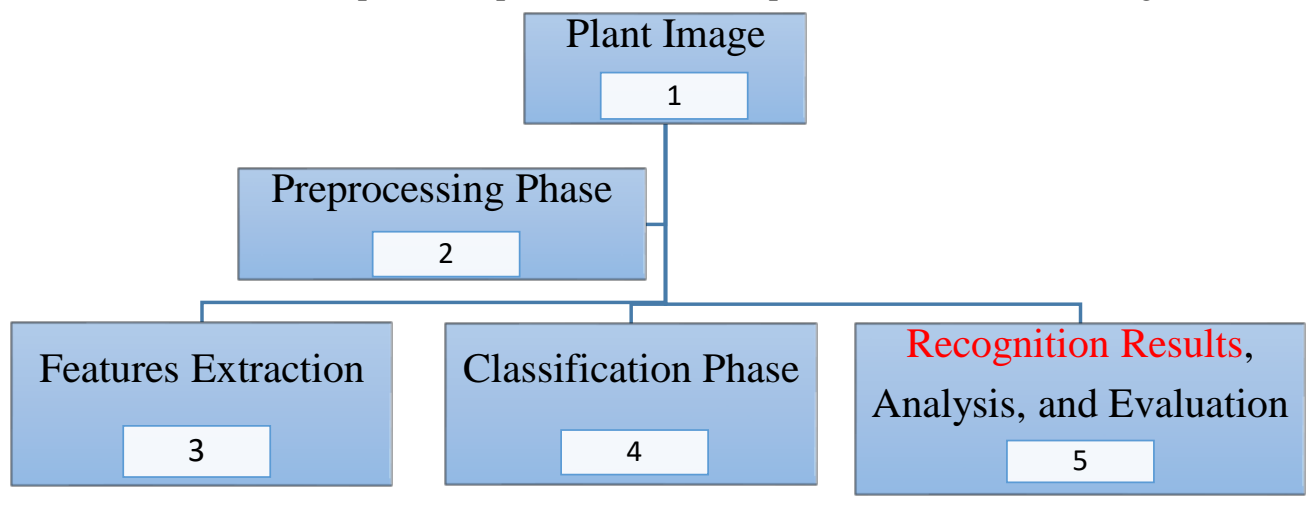

Fig. 2. Plant identification framework phases based on image processing

3. Image-based plant segmentation techniques

Plant image segmentation is based on the method of image acquisition. For example, scanning of separated leaf, photographing a plant leaf, photographing of a separated leaf with the complexity of background. In the segmentation process, isolated leafs that are photographed or scanned on a simple background are similar. In image segmentation process of a plant, the image can be segmented into two groups: plant and background, where background segmentation is an important process. In the literature, there are several methods that had been developed for segmenting the images of the plant. The segmentation methods are threshold-based segmentation, color index-based segmentation, and learning-based segmentation methods.

Image segmentation refers to discriminating objects of interest from their background. Threshold techniques are widely applied in plant detection based on image segmentation. These techniques pose two problems; namely, soil background class and plant vegetation class. Usually, thresholding is applied to transform the original image to determine the class. Choosing the proper threshold plays an important role in the segmentation process. Therefore, if the threshold value is set to be high value, then some important plant pixels may be merged with other background pixels which lead to under-segmentation. While a low threshold that is set to be low may lead to over-segmentation. In the literature, researchers have applied different threshold technique to address these problems. For example, authors in [10] presented a new retrieval method of images to plant identifying by using the features of contour-based shape. Moreover, authors in $[11,12]$ applied shape based leaf image retrieval method and leaf image retrieval with shape features for image retrieval problem.

Segmentation of the regions of the plant from the background consists of stones and soil is considered as an essential part to be performed for the detection of the diseases. Moreover, the performance of the segmentation process can affect the quality of the identified objects from the separated image information. Analyzing the behavior of color indices can provide an approximate understanding of the segmentation performance of the color indices. Their actual qualities cannot be drawn directly from the analysis unless the regions of plant and background are both known for a given context. In the color indices based approach, there are well-known color indices given in the literature which are; the normalized green, the difference between normalized green and normalized red, the normalized difference index, the excessive green index, the modified excessive green index, and the hue. All these indices use color components defined in the RGB or its normalized counterpart RGB space. However, other color models have also been used and tested in the literature. For example, hue, saturation, and intensity (HSI) color triplets transformed from the RGB system were employed to segment tomato plants from weeds [13] and for in-field weed sensing [14].

3. 1. Threshold-based approach

This technique has been applied in plant identification based on image segmentation has two group problems which are soil background class and plant vegetation class. Therefore, to remove the noise in the background of plant images, we can apply the threshold-based technique. In [15], authors presented a new method for threshold selection from gray-level histograms automatically. This method had been improved in [16] by using two-dimension Otsu method. Threshold-based additive Boolean update function attached to each element for the signaling network of a plant has been presented in [17].

\section{2. Color index approaches}

To discriminate plants from the background, color is used as one of the most common methods for this purpose. Therefore, color distribution depends on color intensity, standard deviation, and skewness. Moreover, there are proposed image descriptors in the literature are Color Coherent Vector (CCV) [18], image indexing using Auto Color 
Correlogram (ACC) [19], image retrieval method based on border/interior pixel classification [20], color indexing using GCH (Global Color Histogram) [21].

\subsection{Learning based-approach}

In some situations where we cannot perform the color-based approach in overcast and sunny conditions. Several studies have applied different approaches which include supervised and unsupervised machine learning methods with the transformation of color features. The classification process is based on the classifiers of machine learning such as Support Vector Machine (SVM), decision tree (DT), Naïve Bayes (NB), Naïve Bayes Tree (NBT). A comparative analysis of the leaf image recognition has been presented in [22] by using SVM classifier with $95.47 \%$ accuracy results. An expert system of plant identification has been proposed in [23] to identify different species of plant based on their images of leaves. In this system, the ant colony optimization algorithm is used for feature selection. The efficiency of this system was tested, and the average accuracy was $95.53 \%$. A comparison of the recent method for the recognition of different plant species is desribed in [24].

\subsection{Plant images data sets}

In the last decade, there are multiple plant data sets are available for an attempt toward an electronic field guide for plants of researchers and specialists such as Smithsonian dataset [25], Swedish dataset for the classification process of leaves [26], ICL leaf dataset [27], and others. However, there is a problem of image datasets available for the implementation in plant identification. So far, there are a few number of used datasets are accessible to the scientific community or very limited. Currently, there exist some databases are available, such as the database developed in [28] to enable the development of mobile disease diagnostics through machine learning, in which roughly 53,000 images of healthy and diseased plants are available at (https://www.plantvillage.org/). Also, most of the exists data sets that are used in implementation in most of all researchers are collected manually using modern Cameras and images are captured by researchers themselves such as in CLEF conference in 201171 species of plant in 2011 that are increased to be 126 species in 2012 The datasets were collected in different categories based on scanned images of a leaf, scanlike photographs of a leaf and unconstrained photographs of plant leaf. In some cases, the image database can be created based on microscopes. The images of tomato plant that are introduced in this paper are captured from a farm by authors themselves. The implementation using Matlab toolbox of the preprocessing phase on tomato leaves plant is presented in Figure 3. This phase has been implemented according to the following steps.

1. Images Capturing. In this phase, SVM has been employed in this phase. In the acquisition process, we carefully selected images to achieve the classification process. The used images in this phase are captured to the infected leaves of tomato with two diseases, Powdery mildew, and early blight, from different farms. This real data set includes 200 infected leaves images were 100 for each virus type has been extracted.

2. Images Preprocessing. The main aim of this phase is removing noise in the image and enhancing smoothness that can be caused by the capturing conditions and environment. Therefore, we must isolate and extract each leaf in all images.

3. Image Resizing. Captured images are with different sizes and then these images resized to $512 \times 512$ resolution to reduce the size.

4. Background removal: In the background of images, a shadow may disturb the feature extraction phase. Therefore, the background of each image should be removed by using subtraction technique with some morphological operations.

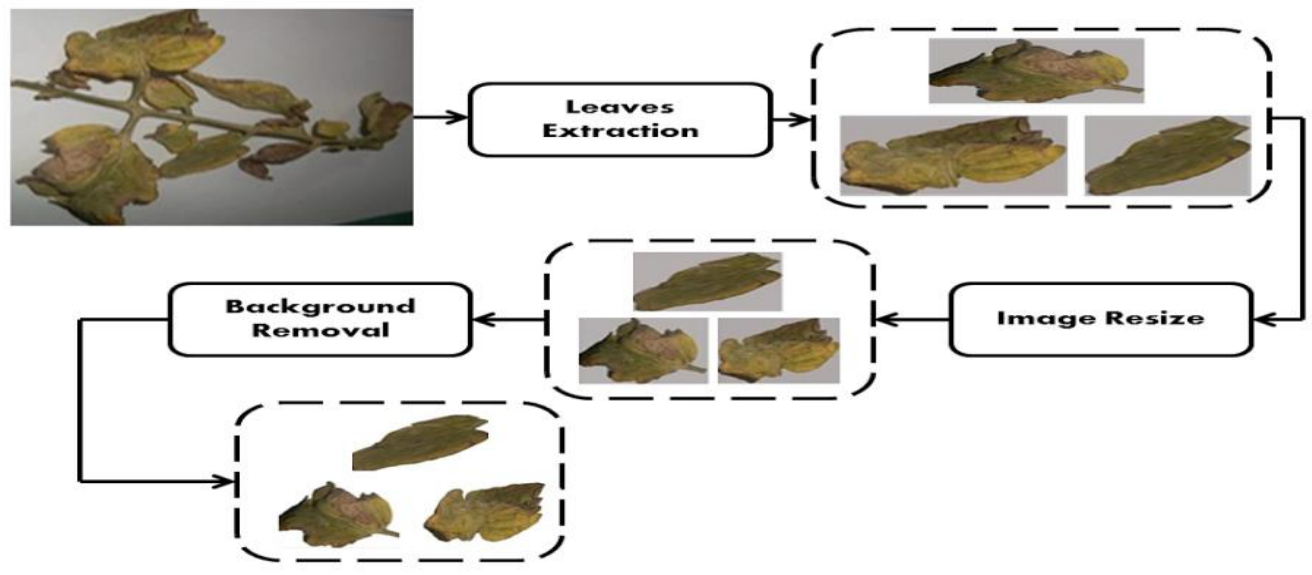

Fig. 3. Preprocessing phase of tomato leaves plant

To analyze the results of this phase, the quality of the images has been enhanced by removing the noise that caused by defects of camera flash or high lights to increase the efficiency of the classification and prediction process. 
Therefore, every leaf has been isolated with extract in the single image. Then, the captured images were resized to 512 x 512 resolution to minimize the storage capacity and reduce the computational time in the post-processing. Finally, the background of each image has been removed by using the background subtraction technique with some morphological operations. Gaussian Mixture-based Background/ Foreground Segmentation algorithm was used to subtract the background and morphological techniques (dilation followed by erosion) to remove noise.

\section{Feature extraction in plant diseases}

Feature extraction is an important stage in pattern classification. Nowadays, the datasets of plant images contain a lot number of features which sometimes are irrelevant or with the high dimension which can affect the performance of accuracy and effect of the results. Therefore, feature selection has been proposed in the last few years to improve the results of the used algorithms and techniques in plant diseases identification. The main objective of the feature selection process is to identify the most important features in the datasets for the optimization of the problems. Identifying the important subsets of the features is considered as important features in reducing the dimension of data to be processed in the classification process. Each image in a given dataset has its features and can be discriminated than other images by some features such as color, brightness, edge, and texture, etc. Other features such as histogram some can be obtained after processing.

Feature extraction is the most important stage of the diseases identification process in plants and segmentation process. For example, a new proposed method of the segmentation of the weed leaf by using deformable templates has been proposed in [29]. While a leaf extraction from complicated background is presented in [30], and a hybrid algorithm for leaf recognition and characterization of images is presented in [31].

\subsection{Shape Feature}

Shape feature is considered as an important feature in plant images, and the extraction process of shape features usually depends on the segmentation of the image. The region of interest (ROI) and the boundary of an object can be segmented.

In this scenario, the shape feature is divided into two groups: features based on regions and the boundary based features. Recently, researchers are applying the integration of shape, color, and texture features to improve the segmentation process.

For example, authors in [32] classified plant leaves based on their texture features using Gabor features and LBP. Authors in [33] applied an integrated system which depends on using texture and shape features, while discrete wavelet transform (DWT) is applied to texture feature. Recognition of plant leaves using support vector machine has been presented in [34]. A shape classification method has been proposed in [35]. A new method for plant identification is presented in [36] based on multiscale fractal dimensions to identify the problem of leaf identification in the plant. Also, another leaf classification method is proposed in [37] based on a supervised method by using label propagation. 4.2. Texture Feature

The texture of an image is represented by the gray scale, and color change of the image is composed of many close elements. Therefore the image texture describes the features of sparse, smooth, and so on. In the literature, the texture of an image can be classified into three methods which are structure method, statistics method, and frequency method. Usually, the texture is often overshadowed by the shape as the dominant for leaf recognition for example, in plant identification disease. The best texture descriptors in the literature are the Gabor features and local binary patterns and fractal dimensions [38]. There are state-of-the-art texture description methods which are introduced morphological texture descriptors as in $[39,40]$.

\subsection{Color Feature}

Color features are considered as visual features which are reliable and stable. The color is not discriminative as texture or shape in recognition of leaves, where plants usually are located in uncontrolled environmental conditions such as the shade of green. Moreover, the colors of leaves depend on the environmental conditions and the season of plants. Also, color is an important factor and has its contribution to the identification of plant diseases. For instance, authors in [41] proposed color descriptors such as RGB histogram, and LSH histogram and color moments [42].

\section{Imaging Classifiers for Plant Diseases Identification}

Classification is an important process in data mining. A classifier uses the input datasets to build classification models. Classifiers usually are used for predicting data sets with binary classes. Using robust and efficient features are representing an important element in the classification process.

\section{Support Vector Machine (SVM) Classifier}

SVM classifier is widely used in different cases as in linear separable and linear inseparable by using the strategy of nonlinear mapping algorithm. The SVM objective is based on dividing the training set into two classes. The main idea of SVM is to map the original data into a high-dimensional feature and construct a hyper plane as the discriminative surface between the negative and positive data. SVM is used to solve the regression and classification 
problems and in bioinformatics problems in different areas such as identifying the plant promoters [43]. SVM classifier is used in the literature to evaluate the effectiveness of each feature in groups and individually, during the implementation process. For evaluation process, authors used the remaining samples from the training set. Automation of plant cell detection process is an important to obtain the development information of individual plant cells to provide tools of research to ease the search for special events, such as cell division. SVM is used to select the cells based on the cell descriptor constructed from the edge strength and shape [44]. Authors in [45] designed an automated system to classify the wheat grains, and the results showed a high level of accuracy. SVM has been applied to differentiate three categories of tea plant; green, black and Oolong teas [46]. Herbal plant images are used in an automatic identification system as in [47] and SVM classifier is used to classify the herbal plants of licorice and rhubarb.

SVM classifier was implemented for an automated system of leaf plant recognition in [48] with an average accuracy of $95 \%$. In this system, the proposed plant leaf classification technique used the SVM classifier that based on shape descriptor, and the rate of recognition was up to $95.16 \%$. Authors in this work presented a method of plant species recognition using SVM for Flavia dataset. In the literature, there are other related models of plant identification and recognition based on leaves images as proposed in [49-53].

\section{Artificial Neural Network (ANN)}

ANN was used to train data set samples (Flavia and ICL) for the automatic classification of plants based on their leaves of a large number of different fruit trees and showed high level of accuracy [54]. Convolutional neural network $(\mathrm{CNN})$ is used in some applications using Android mobile application to classify the natural images of leaves [55]. ANN was used in [56] to study the method of leaf identification with k-Nearest Neighbor (KNN) classifier with accepted recognition rate. ANN is used in leaf recognition with Probabilistic Neural Network (PNN) classifier for different classes of plant leaves samples, and the results showed high recognition rate of $93.08 \%$ [57].

2. $k$-Nearest Neighbor Classifier (KNN)

$\mathrm{KNN}$ is data mining classification techniques which is based on the closest training examples in the feature space can be represented by its closest $\mathrm{K}$ neighbors. A new method for plant leaves classification based on two-dimensional shape feature are proposed in [58]. In KNN, the classification of the selected data set can be performed by taking into account distances between data values of each class. In this context, the plant leaves classification, KNN classifier has been used to classify the plant species using a set of reduced leaf shape feature vectors. Leaf features are employed and used in different plants classification based on KNN classifier. Authors in [59,60] employed KNN to discuss the leaf plant classification and the experimental results showed high recognition rate. A new proposed approach for leaf classification using local features of plant species from low quality pictures using mobile devices is presented in [61]. With the consideration of both local features and global features, new proposed classification methods are used to improve leaf image classification process in $[62,63]$. The $\mathrm{KNN}$ classifier is used in plant identification and recognition using plant images as in [64-66]. In [67] a new strategy of multi-classification system of wheat leaves diseases is proposed with recognition accuracy of $87.13 \%$ by using the KNN classifier. Zernike moments and morphological features are employed with KNN classifier to study classification of plant leaves with a reasonable level of accuracy $[68,69]$. KNN classifier is used in ANFIS (Adaptive Neuro-Fuzzy Inference System) and this system was trained by different leaves of a plant with accuracy ratio is $80 \%$ [70]. Authors in [71] used the geometrical feature to identify plant leaves based on KNN classifier with recognition rate is $93.17 \%$. Authors in [72] used KNN classifier to propose a new approach to classify the plant leaves by using texture features. In order to improve the performance of plant leaf identification, the Fuzzy KNN classifier is introduced in [73].

\section{Probabilistic Neural Network Classifier (PNN)}

PNN is considered as a category of ANN which is based on the statistical principle. Classification can be performed by localized networks such as PNN. PNN classifier was proposed by Specht in 1990 [74]. PNN was based on the computing of Bayes statistics which consists of four layers input layer, pattern layer, summation layer and decision layer [75]. PNN classifier is used to classify leaf plants and was showed a high accuracy as in [76, 77]. Shape feature is applied to discuss the plant identification leaves with PNN classifier as in [78] with accepted recognition rate more than $85.6 \%$. In addition, PNN classifier is used in plant leaves classification and recognition based on the center distance for leaf identification and the recognition rate was about $80 \%$ [79]. Moreover, PNN classifier is used in different areas of plant classification and recognition as in [80,81]. A huge number of plant images from different plant species was taken from Flavia data set is trained by using PNN. The methods which are based on half leaf features showed good results when using PNN, and the results showed high recognition accuracy as in [82, 83].

\section{Hyper Sphere Classifier}


Hyper sphere classifier has the ability to compress the sample data to reduce the space of storage. The main idea of using hyper sphere classifier is to use a hyper sphere represent the points of the cluster. In order to reduce the high dimension of the space, we can use a series of hyper spheres. The overall idea with hyper sphere classifier is based on the approximation of the number of hyper spheres for each sample with the extension of the radius of the hyper sphere and eventually we can use multi sample space and all the sample points. A new classification method based on hyper sphere classifier is presented with accepted level of accuracy in [84].

\section{Back Propagation (BP) Classifier}

$\mathrm{BP}$ is a supervised learning classifier which can compute some functional relationships between its input and output. BP is a multilayer network, fully connected, feedforward network. The first layer is represented by the input while the second layer is represented by the output layer. The hidden layers are defined to be the layers between the input and output layers. Authors in [85] used ANN in the classification process to identify the apple orchard pests. The resulting neural model has been trained by the use of the BP method. A new proposed method to classify the images of plant based on the BP classifier is presented in [86] with a classification rate of $92.7 \%$. The BP is also used to classify the leaves of the plant [87], and the experimental results showed high accuracy level.

6. Random forest $(R F)$ Classifier

The main idea of RF is to build a forest with a random way. The forest consists of decision-making trees. The tasks that are provided by the human experts are useful in Machine Learning applications by using the knowledge about these tasks in the plant classification process as the in case of the veins leaf which need the knowledge of experts [88]. Authors in [89] applied RF algorithm in classification process for LC-MS species of plant species identification. The experimental results showed that the RF is a robust classifier with noisy problems with handling large datasets in fields such as computational biology. A new system of plant identification based on RF is used in [90] with accuracy rate is $88.82 \%$. Moreover, the RF was employed to plant recognition in a complex background in [91] and the accuracy was up to $97.3 \%$. Recently, DNA barcoding as in $[92,93]$ is used in taxonomic research applications. DNA sequence is used to identify of species to which a plant, animal or fungus are belonging.

7. k-Means Classifier

The k-means clustering algorithm is used as iterative process to divide the data set into another classes in order make the evaluation of clustering technique to provide an optimal performance. Therefore, it represents the mean of all data in the clustering process. However, this classifier is not adequate to process a discrete data, on the other hand it has good results with continuous data. Authors in [94] applied k-means classifier in order to identify the plant diseases but the experimental results showed low performance. A new method for plant identification based on using the kMeans classifier is used is used in the literature with an accepted classification rate.

\section{Hybrid Classifiers}

In Machine Learning and Data Mining approaches, hybrid classifiers can integrate the results from multiple algorithms with the aim of improving classification accuracy and algorithms performance. There are some commonly used hybrid classifiers that shows good performance and results with the continuous development of plant identification. In addition, plant genes can be employed as alternative mechanism to explore the important plant functions such as the response to the stress. Therefore, authors in [95].proposed a novel computational method for the identification of plant based on hybrid feature system based on combing the (PWM) position weight matrix with the (ID) increment of diversity. In this system, SVM was used to classify constitutive and alternative splice sites. Numerical taxonomy is considered as a method of data mining in the field of botany. In [96], a particle swarm optimization-aided fuzzy cloud classifier is used based on attribute similarity for plant taxonomy. Plant leaves are classified in [97] by using Linear Discriminant Classifier (LDC).

\section{Current Limitations and Challenges based Image Processing Techniques}

The main objective of this section is to highlight the most crucial limitations and challenges in plant diseases identification to evaluate the performance of the current proposed methods techniques in the literature based on image processing approach.

A. Current problems

Although a lot of methods of plant recognition and diseases detection based on the image were proposed in the last few years, and some of them have achieved recognition results, some problems still exist and summarized in the following.

In the literature review, there are some research studies of the automatic plant identification and its diseases based on image processing techniques. From the analysis process of the current status, there is a gap between the current capabilities of image processing techniques for automatic plant disease identification and the real-world needs Moreover, most of the used image processing techniques are need more investigations and research to deal with a 
wide variety of plant species and diseases identifications of pants. The main challenges and problems in applying image-based methods for plant and its diseases identification can be grouped in the images capturing conditions, the similar symptoms of different diseases, and the noise in the background of images which represent a critical issue and have an impact on performance of the used and applied techniques and methods for plant and its diseases identification. There are some other challenges that affect automatic disease identification that cannot be categorized together with those already discussed such as real-time operation and differences between the distributions of the training data used to learn the model and the data on which the model is to be applied. More researches are needed to focus on new methods for disease identification, for example, based on color transformations, color histograms, pairwise-based classification system, and clustering algorithms.

When we propose new features for feature selection method, we should pay attention to whether it is easy or not to extract these features. With the change of time and the impact of the climate change, the shape or color of the pieces of the plant maybe changes even for the same plant. Therefore, this problem should be taken into consideration in the future studies.

The existing classifiers are not designed especially for plant diseases identification. Therefore using such classifiers in classification process of plant diseases recognition, they show low accuracy and performance rates.

The researchers are using different data sets, and the size of the data sample is also different in each dataset. It is difficult to evaluate the performance of different algorithms or the classifier.

B. Limitations and challenges

In the next, we provide an analysis of each one of the proposed challenges, emphasizing both the problems that they may cause and how they may have potentially affected the techniques proposed in the literature. The list of these challenges is described below.

1. Image background. The segmentation of the ROI (region of interest) for the symptoms of images is usually difficult because of the elements that exist in the background. For instance, sometimes the background of leaves images is noisy with plants or soil or any other elements, then, to segment the ROI this will be considered as a challenge. Therefore, in the last years, the segmentation process of the leaves of plants from a busy background gained a great attention. For instance, Figure 4 shows how the complex background in the image represents a critical challenge.

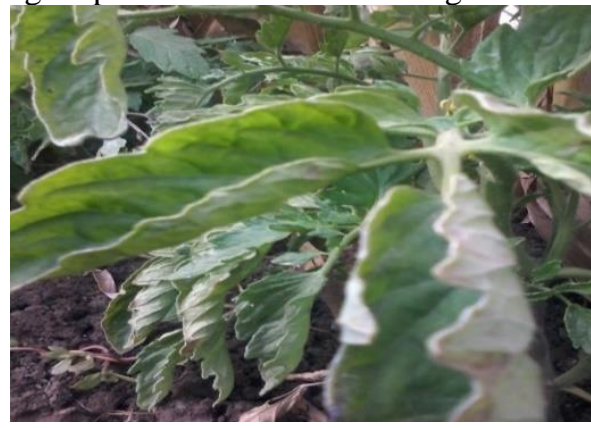

(a)

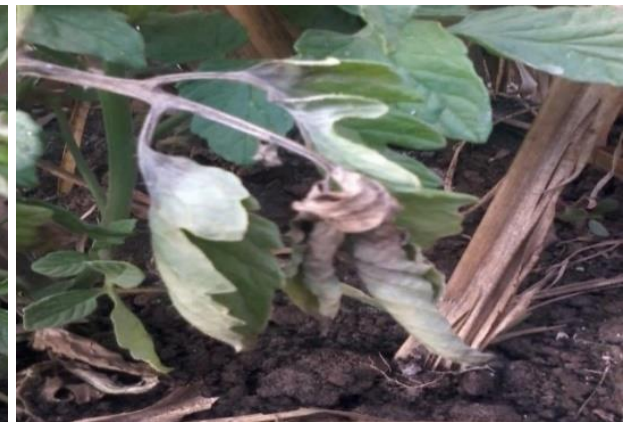

(b)

Fig. 4. Complex image background: (a) and (b) are multi leaves of tomato plant images

2. Image capture conditions. The conditions of capturing plant images are sometimes uncontrolled which can cause difficulty in predicting the characteristics of such images. Also, in practice images have to be captured in the same conditions. Illumination issues are important, where issues like the position of the leaf concerning the sun and overcast conditions. Some of these problems are shown in Figure 5.

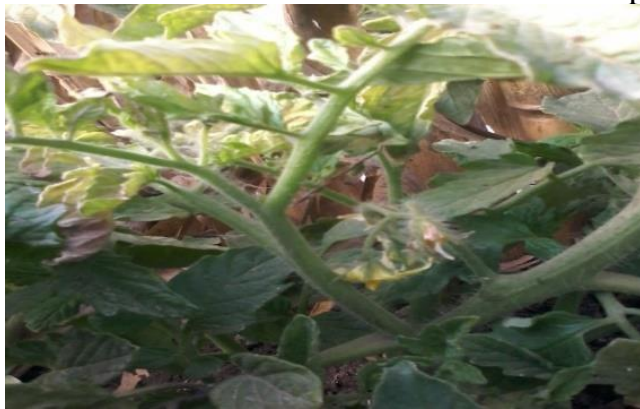

(a)

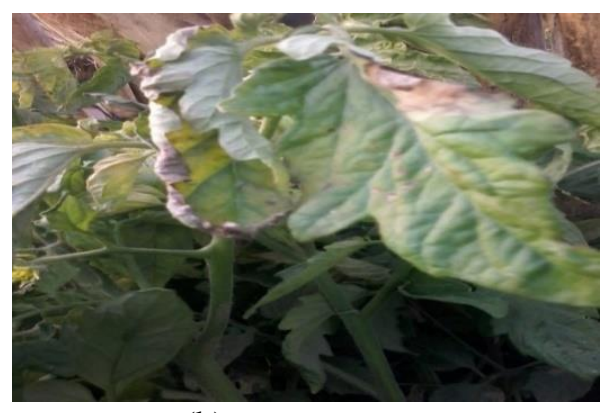

(b)

Fig. 5. Uncontrolled environment: (a) and (b) are tomatoes leaves images under uncontrolled conditions. 
3. Feature extraction. To propose a new feature, we have to take into account whether it is easy to extract or not. With the climate and time change, the shape or color of the plant may be changed. In Figure 6, we can notice that the curve on the leaf is a disease. Moreover, there is another disease on leaf itself which makes the feature extraction is difficult.

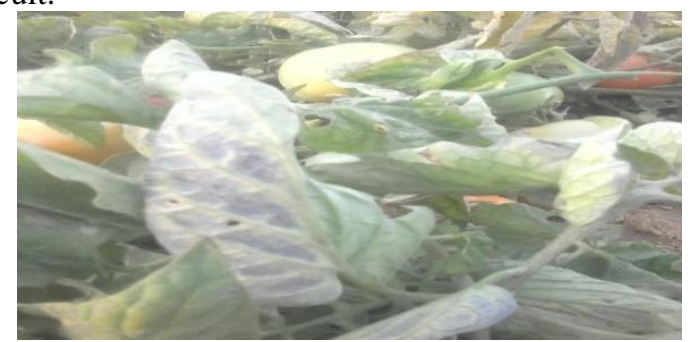

Fig. 6. Tomato leaves image with feature extraction problem

4. Classifiers and algorithms performance. When researchers use datasets, they usually use different size of the data sample from these datasets. Therefore, there is difficulty in evaluating the efficiency of the used classifiers and algorithms for plant recognition.

5. Symptom segmentation. In some cases of diseases, the symptoms on images do not have defined edges or boundaries which make it difficult to identify. It is important to notice that any change in the boundaries of the ROI may impact on the extracted features to describe those regions. The problem of symptom segmentation lacks the solutions and need more investigations and research in the future. The symptom problems are described in Figure 7.

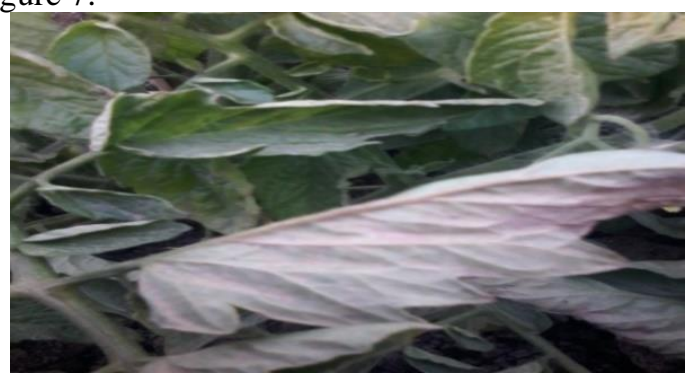

Fig. 7. Tomato leaves image with undefined images

6. Diseases characteristics. A disease may possess very different characteristics and where it exists on the images of the plant.

7. Symptom variations. Different diseases propose different symptoms that may be combined with a hybrid symptom or visually similar that can be difficult to identify among them. Figure 8 depicts the similarity of symptoms of infected leaves.

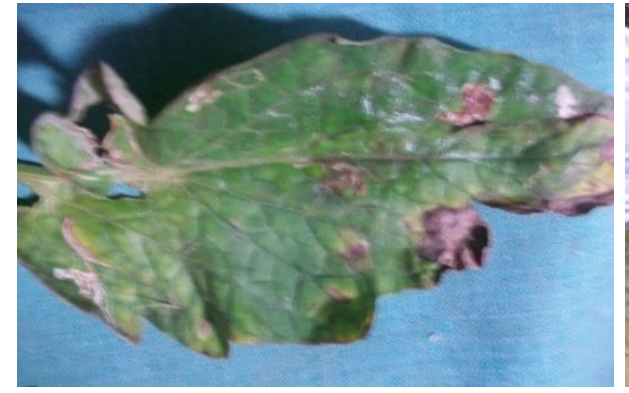

(a)

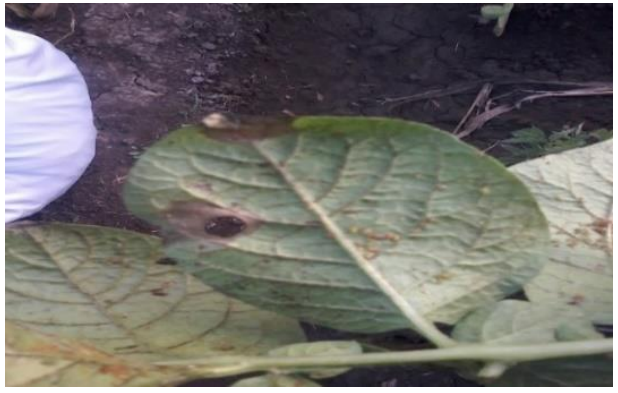

(b)

Fig.8. Similar symptoms: (a) and (b) are infected tomato leaves images with many diseases.

8. Similar symptoms with different disorders. The Similarity of symptoms with different disorders is considered as one of the most important problems in the automatic plant disease diagnosis process.

9. Training data and model data. In the automatic plant identification and the plant diseases, there are differences between the distributions of the used data in the training phase than that are used to the data on which the model is to be applied.

10. Datasets availability. There is few existing databases are used in the implementation phase of plant identification. Moreover, a kind of plant collected in the available datasets is less and not representative for 
deep processing. Moreover, researchers try to collect their datasets themselves which usually do not have a unified rule. As a result, standard benchmark data sets are still challenges.

11. Fully automated systems. In some situations, we cannot fully deal with automatic systems that are based on image processing and computer vision methods and algorithms. Plant agricultural engineers and pathologists often have to resort to resources to obtain a reasonable diagnosis of plant diseases. Therefore, diagnosis system should include various modules which have the capability of providing more information about the current problems. This situation can lead a loss of full automation process. However, additional information will be available for a reliable diagnosis.

12. Real-time Monitoring. Monitoring the plant in the real time will provide many efforts and ease the diagnosis process. Some applications require real time monitoring, while all the proposed systems yet lack the monitoring in the real time. In the future, monitoring is expected to be a proposing solution of the plant diseases identification.

The use of digital image processing in agriculture is quickly becoming ubiquitous, as emulating human visual capabilities is a fundamental step towards the automation of processes. Creating a computer vision system to perform disease diagnosis and severity measurement is one of the most challenging tasks currently underway [98].

\section{Conclusions and Future Work}

Plant diseases cause a major impact on the economics of countries in the industrial agricultural worldwide. Nowadays, computer-based methods of plant identification and plant diseases diagnosis is a new research area, with some problems that still need to be solved. Plant science specialists and engineers can depend on the automated diagnosis systems to identify the plant diseases and recognition. The use of digital image processing and computer vision in plant diseases identification and recognition has shown a great importance in identifying plant diseases in the last years, however there are many limitations and challenges that are described in this paper that need to be overcome by solving and minimizing some of the problems that have been mentioned in this research. Also, with the availability of the advances in image processing, strategies, and algorithms that are applied in the last years. With the developments in image processing techniques which can provide a low cost and propose new advancements in plant diseases identification and recognition. As a result, using digital images through image processing area will be more effective representation method in this problem. This paper reviews the state-of-art of the used techniques, methods, and classifiers that are currently used in the literature for plant diseases identification and recognition. Also, limitations, challenges, and future trends are presented in this area.

To overcome some of the proposed limitations shortly in plant recognition and diseases identification field we need to place constraints on capture images condition. However, this strategy needs more additional efforts which are needed to meet those constraints. In the future, machine learning and computer vision techniques such as Markov Random Fields, Graph Theory, Mean Shift, Deep Learning, and Large Margin Nearest Neighbor classification have to be properly explored and used. Moreover, hybrid system techniques will play an important role in plant recognition and diseases identification. Also, plant specialists and experts and plant sciences will depend on the automatic imagebased systems to support the decision making of the expertise of human.

Nowadays, scouting is the used method for plant monitoring the stress in trees, for example, but it is an expensive method, time-consuming process, and labor-intensive. In the near future, thanks to the advances in technology as Sensor Networks (SNs), Internet of Things (IoT), and Cloud Computing and their integration this can meet real-time requirements of monitoring would be expected to be easier and available with time. Therefore, there is a necessity to develop reliable plant monitoring systems based on sensors, RFID, etc. that would facilitate advancements and assist in monitoring health of plants and plants identifications and recognition.

\section{Acknowledgement}

The author would like to acknowledge the valuable comments and suggestions of reviewers, which have improved the quality of the paper.

\section{References}

[1] Z. Wang, Huale Li1, Ying Zhu, TianFang Xu, Review of Plant Identification Based on Image Processing, Arch Computat Methods Eng, CIMNE, Barcelona, Spain, DOI 10.1007/s11831-016-9181-4, (2016).

[2] H. Goëau, Bonnet, P., Joly, A., Boujemaa, N., Barthelemy, D., Molino, J., Birnbaum, P., Mouysset, E., Picard, M.: The CLEF 2011 plant image classification task. In: CLEF 2011 Working Notes, Amsterdam, (2011).

[3] V. Bakic, Yahiaoui, I., Mouine, S., Litayem, S., Ouertani, W., Verroust-Blondet, A., Goëau, H., Joly, A.: Inria IMEDIA2's participation at Image CLEF 2012 plant identification task. In: Proceeding of CLEF 2012 Labs and Workshop, Notebook Papers. Rome, Italy, (2012).

[4] S. Paris, Halkias, X., Glotin, H: Participation of LSIS/DYNI to Image CLEF 2012 plant images classification task. In: Proceeding of CLEF 2012 Labs and Workshop, Notebook Papers. Rome, Italy, (2012). 
[5] M.E. Nilsback, Zisserman, A.: Delving deeper into the whorl of flower segmentation. ImageVis.Comput. 28(6), 1049-1062, (2009).

[6] B. Yanikoglu, E. Aptoula, C. Tirkaz, Automatic plant identification from photographs, Machine Vision and Applications, 25:1369-1383, DOI 10.1007/s00138-014-0612-7, (2014).

[7] F. Lin, Zheng, C., Wang, X., Man, Q.: Multiple classifications of plant leaves based on Gabor transform and LBP operator. In: Communications in Computer and Information Science, pp. 432-439, Shanghai, (2008).

[8] D. Casanova, Florindo, J.B., Gonçalves, W.N., Bruno, O.M.: IFSC/USP at Image CLEF 2012: plant identification task. In: Proceeding of CLEF 2012 Labs and Workshop, Notebook Papers. Rome, Italy, (2012).

[9] P. Prusinkiewicz, \& Lindenmayer, A. (1990). The algorithmic beauty of plants. New York, NY, USA: SpringerVerlag New York, Inc.

[10] I. Yahiaoui, Hervé, N., Boujemaa, N.: Shape-based image retrieval in botanical collections. In: Pacific-Rim Conference on Multimedia, pp. 357-364, Hangzhou, (2006).

[11] Z. Wang, Chi, Z., Feng, D.: Shape based leaf image retrieval. IEE Proc. Vis. Image Signal Process. 150(1), 3443, (2003).

[12] Z. Wang, Chi, Z., Feng, D., Wang, Q. Leaf image retrieval with shape features. In: Proceedings of the International Conference on Advances in Visual Information Systems, pp. 477-487, London, (2000).

[13] M. R. Golzarian, M.-K. Lee, J. M. A. Desbiolles, Evaluation of Color Indices for Improved Segmentation of Plant Images, American Society of Agricultural and Biological Engineers ISSN 2151-0032, Vol. 55(1): 261-273, (2012).

[14] L. L, Tang. Tian, and B. L. Steward. Color image segmentation with genetic algorithm for in-field weed sensing. Trails. ASAE 43(4): 1019-1027, (2000).

[15] N. Otsu, A threshold selection method from gray-level histograms. Automatica 11, 23-27, (1975).

[16] L. Jianzhuang, L. Wenqing, Automatic thresholding of gray-level pictures using two-dimension Otsu method. Acta Autom. Sin. 19(1), 101-105, (1993).

[17] C. Darabos, Ferdinando Di Cunto, Marco Tomassini, Jason H. Moore, Paolo Provero, and Mario Giacobini, Validating a Threshold-Based Boolean Model of Regulatory Networks on a Biological Organism, Springer-Verlag Berlin Heidelberg, EvoBIO 2011, LNCS 6623, pp. 59-68, (2011).

[18] G. Pass, R. Zabih, J. Miller, Comparing images using color coherence vectors, in Proceedings of the ACM International Conference on Multimedia (ACM- MM'96), pp. 65-73, (1996).

[19] J. Huang, R. Kumar, M. Mitra, W.J. Zhu, R. Zabih, Image indexing using color correlograms, in: Proceedings of the IEEE International Conference on Computer Vision and Pattern Recognition (CVPR'97), pp. 762-768, (1997).

[20] R.O. Stehling, M.A. Nascimento, A.X. Falc ao, A compact and efficient image retrieval approach based on border/interior pixel classification, in Proceedings of the ACM International Conference on Information and Knowledge Management (CIKM’02), pp. 102-109, 2002.

[21] M.J. Swain, B.H. Ballard, Color indexing, Intl J. Comput Vis 7 (1991) 11-32.

[22] J. K. Hsiao, Kang L-W, Chang C-L, Lin C-Y (2014). Comparative study of leaf image recognition with a novel learning-based approach. Sci Inf Conf (SAI) 2014:389-393.

[23] G. MAJ, Khamis S, Mohammad F, Fariman HJ (2015). Feature decision-making ant colony optimization system for an automated recognition of plant species. Expert Syst Appl 42:2361-2370.

[24] MF. Kazerouni, Schlemper J, Kuhnert K-D (2015). Comparison of modern description methods for the recognition of 32 plant species. Signal Image Process 6:1.

[25] G. Agarwal, Belhumeur, P., Feiner, S., Jacobs, D., Kress, J.W.R., Ramamoorthi, N.B., Dixit, N., Ling, H., Russell, D., Mahajan, R., Shirdhonkar, S., Sunkavalli, K., White, S.: First steps toward an electronic field guide for plants. Taxon 55(3), 597-610, (2006).

[26] O. Söderkvist. Computer vision classification of leaves from Swedish trees. Master's thesis, Linköping University, Linköping, (2001).

[27] The ICL leaf dataset. http://www.intelengine.cn/English/dataset/, (2010). Accessed Mar 2014.

[28] D. P. Hughes, \& Salathe, M. (2015). An open access repository of images on plant health to enable the development of mobile disease diagnostics through machine learning and crowdsourcing. arXiv, 1511.08060.

[29] A.G. Manh, Rabatel, G., Assemat, L., Aldon, M.J.: Weed leaf image segmentation by deformable templates. J. Agric. Eng. Res. 80(2), 139-146, (2001).

[30] X. Tang, Liu, M., Zhao, H., Tao, W.: Leaf extraction from complicated background. In: 2nd International Congress on Image and Signal Processing, pp. 1-5, Tianjin, (2009). 
[31] N. Valliammal, Geethalakshmi, S.N.: Hybrid image segmentation algorithm for leaf recognition and characterization. In: International Conference on Process Automation, Control and Computing, pp. 1-6, Tamilnadu, (2011).

[32] T. Beghin, Cope, J.S., Remagnino, P., Barman, S.: Shape and texture based plant leaf classification. In: Advanced Concepts for Intelligent Visual Systems, pp. 345-353, Sydney, (2010).

[33] A. N. Hussein, Mashohor, S., Saripan, M.I.: A texture-based approach for content based image retrieval system for plant leaves images. In: International Colloquium on Signal Processing and its Applications, pp. 11-14, Penang, (2011).

[34] Q. Man, Zheng, C.,Wang, X., Lin, F.: Recognition of plant leaves using support vector machine. In: Communications in Computer and Information Science, vol. 15, pp. 192-199, Shanghai, (2008).

[35] A.R. Backes, Bruno, O.M.: Shape classification using complex network and multi-scale fractal dimension. Pattern Recogn. Lett. 31(1), 44-51, (2010).

[36] O. M. Bruno Plotze, R.de O., Falvo, M., de Castro, M.: Fractal dimension applied to plant identification. Inf. Sci. 178(12), 2722-2733, (2008).

[37] S. Zhang, Lei, Y., Dong, T., Zhang, X.-P.: Label propagation based supervised locality projection analysis for plant leaf classification. Pattern Recogn. 46(7), 1891-1897, (2013).

[38] F. Lin, Zheng, C., Wang, X., Man, Q.: Multiple classification of plant leaves based on Gabor transform and LBP operator. In: Communications in Computer and Information Science, pp. 432-439, Shanghai, (2008).

[39] E. Aptoula, : Comparative study of moment based parameterization for morphological texture description. J. Vis. Commun. Image Represent. 23(8), 1213-1224, (2012)

[40] E. Aptoula,: Extending morphological covariance. Pattern Recogn. 45(12), 4524-4535, (2012).

[41] A. Hanbury.: Circular statistics applied to color images. In: Computer Vision Winter Workshop, pp. 55-60, Valtice, (2003).

[42] F. Mindru, Tuytelaars, T.,Van Gool, L., Moons, T.: Moment invariants for recognition under changing viewpoint and illumination. Comput. Vis. Image Underst. 94(1-3), 3-27 (2004).

[43] A.K.M. Azad, S. Shahid, N. Noman, H. Lee, Prediction of plant promoters based on hexamers and random triplet pair analysis, Algorithms Mol. Biol. 6, 6-19, (2011).

[44] M. Marcuzzo, Pedro Quelha, Ana Campilho, Ana Maria Mendonça, Aurélio Campilho, Automated Arabidopsis plant root cell segmentation based on SVM classification and region merging, Computers in Biology and Medicine $39,785-793$, (2009).

[45] M. Olgun, Ahmet Okan Onarcan, Kemal Özkan, Sahin Isik,, Okan Sezer, Kurtulus Özgis, Nazife Gözde Ayter, Zekiye Budak Basçiftçi, Murat Ardiç, Onur Koyuncu, Wheat grain classification by using dense SIFT features with SVM classifier, Computers and Electronics in Agriculture 122, 185-190, (2016).

[46] Q. Chen. Jiewen Zhao, C.H. Fang, Dongmei Wang, Feasibility study on identification of green, black and Oolong teas using near-infrared reflectance spectroscopy based on support vector machine (SVM), Spectrochimica Acta Part A 66, 568-574, (2007).

[47] B. Fataniya, Meet Josh, Urmil Modi, Tanish Zaveri, Automatic Identification of Licorice and Rhubarb by Microscopic Image Processing, Procedia Computer Science 58,723 - 730, (2015).

[48] S. Prasad, Kudiri KM, Tripathi RC (2011).Relative sub-image based features for leaf recognition using support vector machine. In: International conference on communication, computing and security, icccs, Odisha, India, pp 343346, (2011).

[49] Z. Zhao, Huang X, Yang G (2015).Plant recognition based on leaf and bark images H. J Comput Inf Syst 11:857864.

[50] Z. Wang, Sun X, Ma Y, Zhang H (2014).Plant recognition based on intersecting cortical model. Int Jt Conf Neural Netw 975-980.

[51] C. Priyankara HA, Withanage DK (2015).Computer assisted plant identification system for Android. In: Moratuwa engineering research conference, pp 148-153.

[52] AT. Quadri, Sirshar M (2015).Leaf recognition system using multi-class kernel support vector machine. Int J Comput Commun Syst Eng (IJCCSE) 2(2):260-263.

[53] Z. Wang, Sun X, Zhang Y, Ying Z, Ma Y (2015).Leaf recognition based on PCNN. Neural Comput Appl 27(4):899-908.

[54] A. Aakif, Khan MF (2015).Automatic classification of plants based on their leaves. Biosyst Eng 139:66-75.

[55] TJ Jassmann, Tashakkori R, Parry RM (2015).Leaf classification utilizing a convolutional neural network. In: SoutheastCon. 
[56] M. Amlekar, Manza RR, Yannawar P, Gaikwad AT (2014).Leaf features based plant classification using artificial neural network. IBMRD's J Manag Res 3:224-232.

[57] A. Kadir, Nugroho LE, Susanto A, Santosa PI (2013).Neural network application on foliage plant identification. arXiv preprint arXiv:1311.5829.

[58] A. Hudaya Muhamad Amin, Asad I. Khan, One-Shot Classification of 2-D Leaf Shapes using Distributed Hierarchical Graph Neuron (DHGN) Scheme with k-NN Classifier, Procedia Computer Science 24, 84 - 96, (2013).

[59] S. Zhang, Feng Y (2010).Plant leaf classification using plant leaves based on rough set. In: 2010 international conference on computer application and system modeling (ICCASM), pp V15- 521-V15-525.

[60] S. Zhang, Lei Y-K (2011).Modified locally linear discriminant embedding for plant leaf recognition. Neurocomputing 74:2284-2290.

[61] S. Fiel, Sablatnig R (2010).Leaf classification using local features. In: Workshop of the Austrian Association for Pattern Recognition, pp 69-74.

[62] Z. Wang, Lu B, Chi Z, Feng D (2011).Leaf image classification with shape context and SIFT descriptors. In: International conference on digital image computing: techniques and applications, pp 650-654.

[63] D. J-X, Zhai C-M, Wang Q-P (2013).Recognition of plant leaf image based on fractal dimension features. Neurocomputing 116:150-156.

[64] D. J-X, Wang X-F, Zhang G-J (2007).Leaf shape based plant species recognition. Appl Math Comput 185:883893.

[65] L. Guan-Lin, Zhan-Hong MA, Wang HG (2012).Image recognition of wheat stripe rust and wheat leaf rust based on support vector machine. J China Agric Univ 17:72-79

[66] BS Bama, Valli SM, Raju S, Kumar VA (2011).Content based leaf image retrieval (CBLIR) using shape, color and texture features. Indian J Comput Sci Eng 2:202-211.

[67] Y. Tian, Zhao C, Lu S, Guo X (2011).Multiple classifier combination for recognition of wheat leaf diseases. Intell Autom Soft Comput 17:519-529.

[68] B. Harish, Hedge A, Venkatesh O, Spoorthy D, Sushma D (2013).Classification of plant leaves using Morphological features and Zernike moments. In: 2013 international conference on advances in computing, communications and informatics (ICACCI), pp 1827-1831.

[69] A. AHM, Khan AI (2013). One-shot classification of 2-D leaf shapes using distributed hierarchical graph neuron (DHGN) scheme with k-NN classifier. Proc Comput Sci 24:84-96.

[70] C. Arunpriya, Thanamani AS (2014). A novel leaf recognition technique for plant classification. Int J Comput Eng Appl 4:42-55.

[71] P, Nidheesh, Rajeev A, Nikesh P (2015).Classification of leaf using geometric features. Int J Eng Res Gen Sci 3(2):1185-1190.

[72] YG Naresh, Nagendraswamy HS (2015).Classification of medicinal plants: an approach using modified LBP with symbolic representation. Neurocomputing 173(2016):1789-1797.

[73] X. Wang, Liang J, Guo F (2014).Feature extraction algorithm based on dual-scale decomposition and local binary descriptors for plant leaf recognition. Digit Signal Proc 34:101-107.

[74] D.F. Specht, Probabilistic neural networks.NeuralNetw.3,109-118, (1990).

[75] J. Ben Ali , Lotfi Saidi , Aymen Mouelhi , Brigitte Chebel-Morello, Farhat Fnaiech, Linear feature selection and classification using PNN and SFAM neural networks for an early on line diagnosis of bearing naturally progressing degradations, Engineering Applications of Artificial Intelligence42,67-81, (2015).

[76] W. SG, Bao FS, Xu EY, Wang Y-X, Chang Y-F, Xiang Q-L (2007).A leaf recognition algorithm for plant classification using probabilistic neural network. In: 2007 IEEE international symposium on signal processing and information technology, pp 11-16.

[77] V. Pednekar, Chavan H (2010).A geometrical feature classified leaf recognition algorithm using probabilistic neural network. In: International conference and workshop on emerging trends in technology, pp 1022-1022.

[78] B. Harish, Hedge A, Venkatesh O, Spoorthy D, Sushma D (2013).Classification of plant leaves using Morphological features and Zernike moments. In: 2013 international conference on advances in computing, communications and informatics (ICACCI), pp 1827-1831.

[79] K. Mahdikhanlou, Ebrahimnezhad H (2014).Plant leaf classification using centroid distance and axis of least inertia method. In: 2014 22nd Iranian conference on electrical engineering (ICEE), pp 1690-1694.

[80] P. Nidheesh, Rajeev A, Nikesh P (2015).Classification of leaf using geometric features. Int J Eng Res Gen Sci 3(2):1185-1190. 
[81] A. Hasim Herdiyeni Y, Douady S (2016).Leaf shape recognition using centroid contour distance. In: IOP conference series: earth and environmental science, p 012002.

[82] C. Uluturk, Ugur A (2012).Recognition of leaves based on morphological features derived from two half-regions. In: 2012 international symposium on innovations in intelligent systems and applications (INISTA), pp 1-4.

[83] A. Kadir, Nugroho LE, Susanto A, Santosa PI (2013).Leaf classification using shape, color, and texture features. arXiv preprint arXiv:1401.4447.

[84] J-X, Du, Wang X-F, Zhang G-J (2007).Leaf shape based plant species recognition. Appl Math Comput 185:883893.

[85] P. Boniecki, K. Koszela, H. Piekarska-Boniecka, J. Weres, M. Zaborowicz, S. Kujawa, A. Majewski, B. Raba, Neural identification of selected apple pests, Computers and Electronics in Agriculture 110, 9-16, (2015).

[86] D. Al Bashish, Braik M, Bani-Ahmad S (2010).A framework for detection and classification of plant leaf and stem diseases. In: 2010 international conference on signal and image processing, pp 113-118.

[87] S. Abirami, Ramalingam V, Palanivel S (2014).Species classification of aquatic plants using GRNN and BPNN. AI Soc 29:45-52.

[88] G. Larese, Mónica, Bayá, Ariel E., Craviotto, Roque M., Arango, Miriam R., Gallo, Carina, Granitto, Pablo M., 2014b. Multiscale recognition of legume varieties based on leaf venation images. Exp. Syst. Appl. 41 (10), 46384647.

[89] D.V. Nazarenko, P.V.Kharyuk, I.V.Oseledets, I.A.Rodin, O.A.Shpigun, Machine learning for LC-MS medicinal plants identification, Chemometrics and Intelligent Laboratory Systems 156, 174-180, (2016).

[90] E. Elhariri, El-Bendary N, Hassanien AE (2014).Plant classification system based on leaf features. In: 2014 9th international conference on computer engineering and systems (ICCES), pp 271-276.

[91] D. Hall , McCool C, Dayoub F, Sunderhauf N, Upcroft B (2015). Evaluation of features for leaf classification in challenging conditions In: 2015 IEEE winter conference on applications of computer vision (WACV). IEEE Computer Society, pp 797-804.

[92] P.D.N. Hebert, Cywinska, A., Ball, S.L., DeWaard, J., 2003. Biological identifications through DNA barcodes. Proc. R. Soc. B 270, 313-321.

[93] W.J. Kress, Erickson, D.L., 2007. A two-locus global DNA barcode for land plants: the coding rbcL gene complements the non-coding trnH-psbA spacer region. PLoS One 2, e508.

[94] O. Kruse, Prats-Montalbán JM, Indahl UG, Kvaal K, Ferrer A, Futsaether CM (2014). Pixel classification methods for identifying and quantifying leaf surface injury from digital images. Comput Electron Agric 108:155165.

[95] C. Ying , Jiuqiang Han, Dexing Zhong, Ruiling Liu, A novel computational method for the identification of plant alternative splice sites, Biochemical and Biophysical Research Communications 431, 221-224, (2013).

[96] L. Hongfei, Erxu Pi, Qiufa Peng, Lanlan Wang, Changjiang Zhang, A particle swarm optimization-aided fuzzy cloud classifier applied for plant numerical taxonomy based on attribute similarity, Expert Systems with Applications 36, 9388-9397, (2009).

[97] C. Kalyoncu, Toygar O” (2014). Geometric leaf classification. Comput Vis Image Underst 133:102-109.

[98] J. Garcia Arnal Barbedo, A review of the main challenges in automatic plant disease identification based on visible range images, Biosystems Engineering 144, 52e60, (2016). 